\title{
Hacia una ciencia del trabajo social. Epistemologías, subalternidad y feminización
}

Towards a science of social work. Epistemologies, subalternity and feminization

Belén Lorente-Molina (mblorente@uma.es) Carrera de Trabajo Social, Universidad de Málaga (Malaga, España) ORCID: 0000-0002-4126-4091

Natalia Luxardo (natalialuxardo@conicet.gov.ar) Carrera de Trabajo Social, Universidad de Buenos Aires (Buenos Aires, Argentina) ORCID: 0000-0002-9304-0110

\begin{abstract}
The aim of the article is to analyse a long-standing challenge in the field of social work related to its historical struggle to be taken seriously as a scientific discipline. The paper identifies a tripartite conceptual framework to guide the discussion of the science of social work. The first part describes debates in contemporary social work about the nature of knowledge, considering that social work has experienced researchers who bring critically important perspectives to bear in national and international venues. The second part focuses on the social work's subordinate academic status within the social sciences and its historical positioning as an applied rather than research-oriented discipline and the impacts relating its power and interdisciplinary asymmetric relations experienced in social work. Finally, the article raises questions about the anonymity of women in the profession's history. In that sense, expertise in care, social help and service to the other, are feminized components of the socio-professional identity of social work and a crucial issue to consider in understanding the discipline's standing among other social sciences. The article argues there is evidence of a large variety of positions within the history of social work concerning issues of scientific production within the field.
\end{abstract}

Key words: social work, science, epistemology, feminization, subalternity.

\section{Resumen}

La construcción de una ciencia del Trabajo Social constituye un debate que ha adquirido intensidad disciplinar en la última década. Ello es resultado de los esfuerzos progresivos de vigilancia y depuración epistemológica que se ha venido produciendo allí dónde el Trabajo Social tiene presencia. Para contribuir a dicho proceso, este artículo propone un vértice analítico organizado en tres momentos. El primero de ellos se adentra en cuestiones epistémicas mostrando las construcciones internas que el Trabajo Social ha venido discutiendo con relación a la naturaleza del conocimiento que produce. El segundo momento incide asuntos relativos al poder y en las relaciones asimétricas interdisciplinares que experimenta el Trabajo Social en las Ciencias Sociales. El tercero introduce la necesidad de atender la feminización del sujeto constructor de la disciplina como parte de su identidad epistémica secular. Se concluye que la autonomía como disciplina científica depende de las comunidades epistémicas que conviven en Trabajo Social puedan 
hacer explícitos sus debates que rescaten saberes ausentes, olvidados, postergados y subalternos en las jerarquías de la sociedad de conocimiento, tanto por cadenas internas como por condicionantes externos que limitan la consolidación del ethos disciplinar.

Palabras clave: trabajo social, ciencia, epistemología, feminización, subalternidad.

\section{Introducción}

Some of our social sciences friends are afraid that we cannot be scientific because we really care about what we are doing (Edith Abbott)

El Trabajo Social no ha estado ajeno a los debates fundamentales que se han venido produciendo en el interior de las ciencias sociales, precisamente la preocupación por el enfoque científico social que pudiese dar cuenta cabalmente de su especificidad lo ha hecho estar atento, vigilante, inconforme y, por supuesto, crítico. En este artículo interesa contribuir a la visibilidad de algunas de sus discusiones, las cuáles padecen ciertas limitaciones para su difusión debido a las condiciones de trabajo existentes en el colectivo profesional y académico o a tener una menor tradición de publicación en revistas indexadas y con impacto con relación a otras ciencias sociales. En cierta forma, las dificultades para su visibilidad han provocado que sus análisis y construcciones internas sean insuficientemente conocidos en otras disciplinas sociales. No obstante, el desconocimiento no ha evitado el encasillamiento en un estatus epistemológico de corto alcance. Por tales circunstancias, con este artículo se intenta evitar, en la medida de lo posible, que desde fuera del Trabajo Social se ofrezcan prescripciones epistemológicas, consejos y otras terapéuticas sin tener en cuenta que en el interior de esta profesión feminizada la ciencia ha sido importante siempre, aun admitiendo las dificultades internas y externas que en relación con el conocimiento social se ha experimentado en este sentido. En cualquier caso, el problema del estatus epistemológico se sabe que no es privativo del Trabajo Social, sino que constituye un dilema identificable en las ciencias sociales en toda su extensión.

Los argumentos que se desarrollan en este artículo responden a una estructura teórica elaborada para analizar el problema planteado a través de vértice analítico que descansa en tres dimensiones. Una de ellas relacionada con cuestiones de naturaleza más estrictamente epistémicas; otra tiene que ver con las relaciones asimétricas que el Trabajo Social experimenta respecto de otras disciplinas, y la tercera hace referencia a la cuestión del sujeto cognoscente. Para cada una se definieron estrategias ad hoc que permitieran dar cuenta de la especificidad de los objetos tratados, pero también para ir delimitando conexiones profundas y menos visibles que entre ellos se produce. Primero, se identificaron diversas epistemologías que definieron posiciones sobre la construcción de conocimiento científico en Trabajo Social. Se realiza con tal fin un metaanálisis cualitativo comparando perspectivas en 100 textos seleccionados a partir de descriptores (trabajo social, investigación, ciencia y epistemología) en distintas bases de datos y publicados en las últimas cuatro décadas en español (50) y en inglés (50) en revistas y/o libros. Se distinguieron posiciones sobre la naturaleza del conocimiento en la disciplina, revisando fundamentos epistemológicos de soporte. Como el interés no era realizar un abordaje histórico exhaustivo, sino identificar la variabilidad de perspectivas existentes, fueron suprimidos/as autores/as cuando las posiciones planteadas ya habían sido expuestas por otro/a.

En un segundo momento se inserta en la discusión la necesidad de pensar la estructura subalterna del Trabajo Social a partir de las relaciones jerarquizadas que viene manteniendo con las ciencias sociales, tanto desde una dimensión interna como externa. Este enfoque permite trascender el hecho de la ventaja 
intrínseca de la interdisciplinariedad, para situarla en un nivel de análisis que muestre el poder que se establece en las relaciones de conocimiento. Se otorga centralidad a la tensión que se produce en el interior de la disciplina sobre el debate teoría-práctica, por ofrecer pistas de envergadura acerca de la conflictiva relación del Trabajo Social con la ciencia, pero sobre todo con el impacto desregulador que la exigencia empírico racional produciría de ser aceptada acríticamente. La otra cara, pero concomitante de este ítem, tiene que ver, de un lado con el juego de movimientos que se observa en el espacio académico en los niveles de maestría y doctorado ajenos a la especificidad del Trabajo Social y en las profesionales que crecientemente participan de ellos, entre otras, debido a los rigores de acreditación universitaria y de competitividad laboral. Y, de otro, con las dinámicas de invisibilidad de los saberes provenientes del Trabajo Social que se producen en esas mismas instancias de posgrado.

La última parte consiste en plantear transversalmente la relevancia que para esta argumentación adquiere la feminización histórica del sujeto constructor de la disciplina. Desde una perspectiva genealógica se ha identificado que las prácticas de cuidado y ayuda social desempeñadas en el ámbito público por mujeres en el Occidente Cristiano permite identificar la existencia de una episteme generizada que está en la base de las modernas profesiones femeninas, situación que viene condicionando tanto su horizonte científico, cómo las posibilidades de visibilidad y reconocimiento de los saberes y prácticas que la configuran. El Trabajo Social cuando debate sobre su especificidad epistemológica se interroga por su identidad disciplinar-profesional. El debate por la identidad no puede ser disociado del sujeto que la produce, pues son sus profesionales los/as que le otorgan sentido y finalidad social.

\section{Antecedentes contemporáneos de un debate global sobre una ciencia del Trabajo Social}

Al examinar antecedentes relativos al estatus que tiene la producción de conocimientos en la disciplina puede delinearse un mapa basado en discusiones epistemológicas que surgieron según momentos históricos y regiones, pero que coexisten de manera solapada, heterogénea, más o menos consistente, mostrando el carácter consolidado o bien pre-paradigmático de estas posiciones. Michalle Barak y John Brekke preguntan en su artículo Social work science and identity formation for doctoral scholars within intellectual communities si el Trabajo Social debe aspirar a este saber científico específico o si, por el contrario, no es esta aspiración erosiva de sus valores históricos, concluyendo que es necesario hacerlo para que otros/as puedan criticar los conocimientos generados desde la disciplina. De otro modo, los que elaboran los cánones en ciencia seguirán siendo siempre externos. En la revisión de la literatura se identifica un relativo consenso con relación a la necesidad de definir las bases para una ciencia del Trabajo Social, posible a través de una intersubjetividad epistémica. Se defiende un trabajo epistemológico explícito en la disciplina tanto en la literatura anglosajona como latinoamericana.

Claudia Mosquera sostiene en Conocimiento científico y saberes de acción en trabajo social que en la conferencia de la National Association of Social Work (NASW) realizada en Estados Unidos en 1949 se adoptó al positivismo lógico como modelo para la investigación y la intervención, adhiriendo así a un modelo técnico-racional científico de la formación con claras resonancias en distintas partes del mundo. Serán identificadas algunas posiciones que le contestaron a este positivismo y que propusieron otros enfoques, tanto en Latinoamérica como en países de América del Norte y de Europa, mostrando el carácter global de este debate que, por supuesto, contiene diferencias culturales y de contextos.

Si bien escapa al recorte temporal del artículo, por su carácter inédito el punto de partida será el movimiento que surge en Latinoamérica a mediados del 60, conocido como la Reconceptualización, definido como una reacción contra la filosofía pragmática y empírica del Trabajo Social de corte 
funcionalista. Gloria Leal y Edgar Malagón (Historia del trabajo social latinoamericano) señalan que es el intento más visible para la construcción de una conciencia epistemológica en el que se redefinen compromisos éticos, metodologías, técnicas y áreas de incumbencia de la disciplina. En las décadas que siguieron académicos del Trabajo Social fueron afianzando perspectivas basadas en posiciones teóricometodológicas de inspiración marxista, como el sólido trabajo de Marilda Villela lamamoto (Servicio social y división del trabajo), cuyas críticas al funcional-positivismo como base de la disciplina se alinearon con las miradas realizadas en otros campos disciplinares. Se denuncia que este enfoque promueve la naturalización de la realidad social y la segmenta, desconociendo su historicidad, que reproduce un orden conservador del sistema capitalista, que prevalece un interés pragmático, mecánico y causalista, que individualiza de los problemas sociales al remitirlos a una problemática singular que los coloca en el plano de lo psicológico, que no sirve para comprender situaciones sociales complejas, ni considera las relaciones de poder, etc.

En la década del 80 desde el seno del Trabajo Social surge una propuesta de producción de conocimientos sobre bases epistemológicas provenientes del constructivismo, la hermenéutica, la fenomenología y el materialismo dialéctico que es conocida como la sistematización de la práctica. Adriana Clemente (Notas sobre investigación, formación y práctica profesional) la define como una metodología de investigación que tiene a la práctica social como objeto de problematización y producción de conocimiento, con sustento en los principios del análisis cualitativo y los estudios micro-sociales. Sugiere Ulises Toledo (Una epistemología del trabajo social) que, si bien fue condición necesaria la sistematización, no fue condición suficiente para constituir las bases de la disciplina, dado que los conocimientos también deben ser validados. Teresa Matus (con base en el pensamiento de la Escuela de Frankfurt) dice que hay que sacar al Trabajo Social del polo binario del acotamiento de la acción reflexiva, ya que desde allí se encuentra encapsulado para trascenderla al no contar con teorías consistentes para traspasar el límite del objeto.

Desde una epistemología constructivista, Natalio Kisnerman (Pensar el trabajo social) defiende la postura del Trabajo Social como disciplina científica en la que la investigación debe ser un instrumento para la emancipación, articulando métodos plurales y participativos implementados localmente, que rescaten las diferentes posiciones de las personas, incluidos los valores del investigador que deberá deconstruir, construir y reconstruir sus propias categorías teóricas. Lorente alude a la noción de "momento epistemológico" para convocar a "la reflexión y a la construcción-deconstrucción de unas prácticas de saber que analice la naturalización de unas determinadas prácticas sociales de conocimiento" (Lorente 2002:7), el cual nos permitiría vernos desde una posición "etic".

Estas perspectivas denuncian que el Trabajo Social sea entendido como una "tecnología" a la que no le correspondería la producción de conocimiento científico, sino la aplicación práctica del acervo teórico generado por "las" ciencias, marcando una separación radical entre disciplinas que producen conocimientos y las que los aplican. Esta relación polarizada ciencia/técnica, teoría/práctica tiene un papel clave en la situación de subalternidad en la que el Trabajo Social es ubicado con relación a otras ciencias sociales. En tal sentido, Miguel Miranda (Trabajo social: la construcción de una disciplina científica), así como Travi en el prólogo al libro de Miranda, analizan el proceso histórico en el que saberes producidos desde la disciplina que fueron negados, silenciados y/o desvalorizados. Puntualizan en los estudios realizados por las pioneras en Trabajo Social en los albores de la Escuela de Chicago y las innovadoras categorías teóricas que abordaron después se pierden y reingresan al Trabajo Social de la mano de otras disciplinas. 
Gloria Leal y Edgar Malagón (Historia del trabajo social latinoamericano) mantienen que la reflexión sobre la historia del Trabajo Social en el contexto latinoamericano tiene que replantear varias incongruencias, tales como desestimar los desarrollos del Trabajo Social antes de 1970 al considerarlos bajo la influencia del ideario católico, asistencialistas y reproductores del establishment y considerar el pensamiento elaborado entre los setenta y los noventa como el verdadero Trabajo Social, porque así la literatura existente sobre la historia de la disciplina no alcanza a leer íntegramente su desarrollo.

Con respecto a lo que ocurre en países anglosajones, en Alemania desde finales de los 80 se producen vigorosas discusiones sobre una ciencia del Trabajo Social, en las que una posición que resalta es el realismo crítico, que denuncia la producción de un conocimiento fragmentado y atomizado característico en la disciplina, como señalan H. Göppner y J. Hämäläinen (Developing a science of social work). Estos autores abogan por una ciencia del Trabajo Social para poder resolver inconsistencias y otorgar coherencia a los conocimientos, además que permitiría afianzar un punto de anclaje para la justificación de las estrategias de intervención de la disciplina, según H. Göppner (Epistemological issues of social work science as a translational action science).

En Estados Unidos, J. S. Brekke (Shaping a science of social work) nota que el Trabajo Social no usa para describirse a sí mismo el término ciencia, volviendo de este modo difícil poder distinguir entonces en qué consistiría una ciencia de la disciplina y ser reconocido por otros, si desde adentro no se lo hace. Considera que el Trabajo Social no tiene una gran producción científica que contribuya a expandir el conocimiento de las ciencias sociales y que traduce de otras disciplinas todo lo que son sus referencias sobre investigación. Concluye que la práctica basada en la evidencia es un buen ejemplo de Trabajo Social científico. H. Soydan (Understanding social work in the history of ideas) acuerda que las intervenciones deben estar basadas sistemáticamente en prácticas de "probada" eficacia derivadas de la investigación empírica, para lograr un Trabajo Social más eficiente, transparente y ético. Ve en la práctica basada en la evidencia la forma científica para saber qué intervenciones son más apropiadas, ya que implican la transferencia explícita de evidencia a la práctica de la disciplina.

E.G. Guerrero (What does it take for social work to evolve to science status?) rescata que en este enfoque basado en la evidencia los resultados de la investigación pasan directamente a la práctica. B.R. Crisp (The challenges in developing cross-national social work curricula) dice que, aunque en la disciplina no se usan experimentos puros, muchas investigaciones siguen la lógica de los ensayos clínicos randomizados y que las críticas que los acusan de reduccionistas o de no ser éticos solo sirven para producir una invisibilidad de la investigación experimental en la disciplina. Este dato lo confirma el estudio de B.A. Thyer (A bibliography of randomized controlled experiments in social work), quien detecta más de 700 ensayos clínicos randomizados realizados por trabajadoras sociales que se perdían en la literatura académica específica de otras disciplinas. [Dada la discusión sobre el papel de la mujer en el Trabajo Social (ver sección 3 del artículo) utilizamos el término "trabajadoras sociales" como un universal que incluye también a los trabajadores sociales varones y así evitar el continuo cambio de voces (las/los)].

Son múltiples las perspectivas que enfrentan a este enfoque. B. Pease (Challenging the dominant paradigm) sostiene que la práctica basada en la evidencia está localizada dentro del paradigma funcionalista y asume premisas positivistas sobre la naturaleza de la realidad. I.A. Reed (Epistemology contextualized) sugiere que el positivismo responde reduciendo los contextos de explicación y de investigación a operaciones de conocimiento que pueden intersubjetivamente acordarse en determinada comunidad epistémica a través de una lógica que implica una racionalidad vía exclusión pero que ignora los valores de investigadores/as. 
L.C. Green (Pariah profession, debased discipline?) acuerda que la apropiación de un modelo gold standard de las ciencias naturales es demasiado simplista para la complejidad y la multi-determinación de los problemas que enfrenta el Trabajo Social, que termina encorsetado en su autonomía profesional, además de considerarla éticamente cuestionable. A. Méndez (Claves epistemológicas para el trabajo social) advierten el peligro que las investigaciones en Trabajo Social den por sentados estándares que gozan de mayor consenso, pero que son insuficientes para dar cuenta de aspectos propios de la disciplina, como los saberes tácitos. Desde la hermenéutica, W. Lorenz (Hermeneutics and accountable practice) nota que el cambio hacia paradigmas de investigación basado en la evidencia se relaciona con el avance de políticas neoliberales que reestructuran los modelos de estado de bienestar.

P. Sommerfeld (Social work as an action science) entiende que el Trabajo Social es una ciencia de la acción o ciencia aplicada, que transforma las acciones/intervenciones en problemas cognitivos basándose en un modelo de Bunge a través del cual explica por qué es también una tecnología, pero no solamente una tecnología. Nota una fragmentación de saberes en su producción. Señala que este distanciamiento de la acción inmediata es el primer principio para una ciencia, ya que es el que permite tener tiempo para observar el fenómeno en perspectiva y explicarlo. S. Houston (Reviewing the coming crisis in social work) y otros autores alineados en el realismo crítico creen que no hay razón para rechazar ningún método, sino que plantea la necesidad de un pluralismo metodológico y del pragmatismo que debe prevalecer ante al puritanismo ortodoxo. M. Mäntysaari (Realism as a foundation for social work knowledge) retoma las críticas que se hicieron al Trabajo Social de ser seudo-científico y sostiene que no se puede dar cuenta de las estructuras del mundo inductivamente.

Para E.G. Guerrero (What does it take for social work to evolve to science status?), el Trabajo Social está comprometido con mejorar el bienestar de las personas y las distintas posiciones acuerdan en generar conocimiento que sirva para orientar las prácticas y las políticas sociales destinadas a estas poblaciones. Sin embargo, dice que no hay consenso sobre cómo se lo genera. L.C. Green (Pariah profession, debased discipline?) sugiere que el Trabajo Social tiene pobre el registro de sus investigaciones, con escasas discusiones sobre los tipos de investigación que debe realizar, sobre qué valores, de qué modo y qué cuestiones como la sobre-dependencia en las habilidades prácticas -como competencias opuestas a las teóricas- son factores que derivan en un lugar subordinado que ocupa en la academia y en la práctica con relación a otras profesiones, las que no tienen demasiada claridad sobre sus áreas de incumbencia y metodologías. M.E.M. Barak y J.S. Brekke (Social work science and identity formation for doctoral scholars within intellectual communities) brindan evidencia que las trabajadoras sociales hacen poco uso de los resultados de las investigaciones que ellas mismas generan.

J. Longhofer y J. Floersch (Values in a science of social work) señalan que todas las disciplinas sufren de una proliferación de objetos de conocimiento a través del cual se fragmentan en especializaciones, temáticas y técnicas y el Trabajo Social es parte de esta fragmentación, que emerge y existe como una ciencia en crisis. Encuentran en el realismo crítico la mejor manera de conjugar tradicionales antinomias entre teoría y práctica, lo normativo y lo descriptivo, hecho y valor. K. Briar-Lawson (Critical realism. Response to Longhofer and Floersch) se pregunta si tales argumentos son exclusivos del realismo, y no también de otros enfoques como el feminismo y el post-positivismo. Advierte que si bien el realismo crítico considera que la causalidad debe ser buscada en estructuras y mecanismos que no son observables, pierde de ver la capacidad de agencia de las personas y el poder para transformar colectivamente esas estructuras, que nunca están completamente cerradas. 
J.W. Anastas (The science of social work and its relationship to social work practice) revisa en su artículo todas estas distintas epistemologías posibles para una ciencia del Trabajo Social y concluye que el constructivismo no le permite al Trabajo Social establecer parámetros para juzgar criterios de justicia, ya que termina cayendo en una suerte de relativismo en el que todas las posiciones son tratadas como igualmente válidas; rescata del realismo crítico el papel asignado a factores sociales, culturales y estructurales que median nuestro conocimiento sobre el mundo, es decir, la prioridad del contexto; revisa las posiciones del pragmatismo y el uso de métodos comunitarios y democráticos y por último, menciona a los estudios críticos que abarcan desde el feminismo hasta los estudios postcoloniales y su rechazo a categorías homogeneizantes, revalorizando conocimientos tradicionalmente invisibilizados (como los indígenas), denunciando el uso de la ciencia para perpetuar el poder de las clases dominantes. Estos ejes relativos la asimetría de saberes serán abordados en los siguientes puntos.

\section{La controvertida relación del Trabajo Social con la ciencia}

Como viene exponiéndose, la cuestión entre el Trabajo Social y su fundamentación científica constituye una discusión histórica, reconocible desde los inicios de la profesión y recurrente hasta la actualidad. Puede afirmarse que esta discusión evoluciona acompañada de algunos ejes problemáticos, sin detrimento de que pudieran identificarse otros, que se detallan seguidamente. Uno de ellos tiene que ver con la relevancia que la tensión teoría y práctica ocasiona en el mismo desarrollo del Trabajo Social y, por tanto, en los corolarios acerca de la posibilidad de "un programa de investigación sobre su especificidad epistemológica" (Lorente y Zambrano 2016:364). E. Grassi (La producción en investigación social y la actitud investigativa en el trabajo social) reconoce que el Trabajo Social ha tenido una relación conflictiva con el conocimiento expresada en que se ha llevado al extremo una forma dicotómica de pensar la realidad que separa radicalmente realidad-teoría y discurso-acción, ignorando las teorías implícitas a partir de las cuales la trabajadora social "operativa" define una situación como un problema social.

Esa fricción entre la teoría y la práctica nos sitúa, más que en una aproximación reductora a cómo se concibe el problema del conocimiento, en una tensión simbólica más profunda que remite a órdenes opuestos y jerarquizados sobre el conocer y el hacer que debe también indagarse, entre otras, porque dedicarse al conocer posee un reconocimiento social del que no disfruta el hacer. Es una tensión que responde a su vez a la moderna división social y técnica del trabajo que procede separando, jerarquizando, depurando y organizando espacio, evitando mezcolanzas e impurezas. Cabe la pregunta acerca de si la preeminencia adquirida en el Trabajo Social del enfoque basado en la evidencia, sobre todo en el contexto anglosajón, puede responder a una reacción que simboliza la angustia ante el hecho de no tener todavía resuelto cómo operan distintos componentes epistémicos del Trabajo Social bajo las exigencias de la llamada sociedad del conocimiento. Es claro que el camino es largo, pero no se soluciona amputando las partes que no se controlan, ni desde luego es la vía válida para la depuración epistemológica. Hay que sopesar que los saberes de acción se consideran incompletos, difícilmente clasificables e irreducibles a un orden de conocimiento estable.

La complejidad de la estructuración epistémica del Trabajo Social y la consecuente densidad de su objeto constituyen dos dimensiones a las que B. Lorente (2002) apunta para que la producción de conocimiento en Trabajo Social experimente mayores dificultades para ser comprendida bajo el reduccionismo del debate racionalista, en tal sentido sería comprensible que la disciplina no haya hallado el modo de encajar en un corsé que en el fondo limita su expansión. Foucault muestra la interacción constante entre regiones del conocimiento y subraya la complejidad y heterodoxia inherente a la producción de saberes sobre lo social. La amplitud y la interdependencia de estas regiones permiten crear objetos de estudios 
interactuantes, lo que confirma que "todas las ciencias humanas se entrecruzan y pueden interpretarse siempre una a otras, sus fronteras se borran, las disciplinas intermedias y mixtas se multiplican indefinidamente y su objeto propio acaba por disolverse" (Foucault 1986:347).

En Trabajo Social, el asunto de la complejidad aparece con un matiz particular puesto que la acción práctica se debate continuamente con la vida cotidiana, es decir, con el vasto mundo de la reproducción social, pero lo hace enlazándolo necesariamente con las instituciones, la política, las realidades económicas, los derechos y la justicia social, es decir, al vasto mundo de la producción de lo social. Morin señala que la práctica del conocer que hiper-simplifica lo real forma parte de la inteligencia ciega que caracteriza nuestro tiempo y, a su vez, da contenido a lo que define como patología de la razón: "la racionalización que encierra a lo real en un sistema de ideas coherente, pero parcial y unilateral, y que no sabe que una parte de lo real es irracionalizable (no puede racionalizarse), ni que la racionalidad tiene por misión dialogar con lo irracionalizable" (Morin 1994:34). Las tensiones entre lo que puede racionalizarse y lo que no puede serlo, forman parte de los saberes que se producen en la práctica de la intervención social, enmarcado, a su vez, en las diferentes tradiciones epistemológicas existentes en Trabajo Social.

\subsection{De las asimetrías de poder del Trabajo Social en las ciencias sociales}

En concomitancia con la reflexión anterior, el segundo eje al que se hacía referencia tiene que ver con el hecho de que dicha discusión no se ha producido en condiciones de simetría con otras disciplinas de las ciencias sociales, sino bajo interacciones jerarquizadas de conocimiento y con prácticas de tutelaje que puede afectar tanto al ámbito académico como profesional.

Esta perspectiva nos sitúa en la importancia de pensar las ciencias sociales en su complejidad concreta más que en su armonía abstracta, sobre todo porque son más interesantes y reveladoras al ser abordadas como un campo de poder, tal y como lo analiza Bourdieu, que como un espacio neutro en el que se producen una necesaria mixtura del conocimiento e intercambio disciplinar. Una cuestión es el debate sobre cómo el Trabajo Social se relaciona epistemológicamente con otras disciplinas, analizando las bondades de la intersección de saberes, y otra bien distinta son las relaciones de poder existentes al interior del mundo académico, que están también condicionando el modelo asimétrico de interdisciplinariedad que caracteriza al Trabajo Social.

Esta asimetría adquiere una condición singular en la medida que las mismas ciencias sociales se encuentran sometidas a un intenso proceso de desregulación, proceso concomitante a la reorganización post-fordista del trabajo que con dureza han estado padeciendo las profesiones sociales. La demanda sobre qué ciencias sociales se necesitan para la fase capitalista en la que nos encontramos, posee una estrecha relación con algunos discursos engañosos sobre qué podemos llegar a entender por la invocada y socorrida transdisciplinariedad ante la complejidad del mundo actual, como si ésta fuese algo nuevo. Una vez más, siendo conscientes de que el debate transdisciplinario es una oportunidad y una necesidad, también se considera que a tal debate es ineludible entrar desde una identidad epistemológica, si no, la confusión podría incluso acrecentarse dado el rigor que exige ir más allá del discurso de lo transdisciplinar para concretar los privilegios de su utilidad.

A partir de ello, la idea de autonomía no puede ser concebida únicamente en relación con el poder directo que las trabajadoras sociales desempeñan a través de sus decisiones, sino fundamentalmente a los condicionamientos imperceptibles que están influyendo en el posicionamiento del Trabajo Social allí donde tiene presencia. En tal dirección apunta P.S. Nurius (Social work preparation to compete in today's 
scientific marketplace) para el contexto anglosajón, en el que otras disciplinas con una presencia tradicionalmente más visible en el ámbito de la investigación que el Trabajo Social se "sorprendían" cuando académicos de Trabajo Social se desempeñaban como pares, notando los estereotipos y percepciones que aquellos tienen sobre la disciplina, especialmente en contextos de ajuste en políticas científicas para las ciencias sociales. Como nos explica Bourdieu (Los usos sociales de la ciencia), el poder de un campo se afianza en función del grado de autonomía que pueden conquistar, o se resquebraja por la influencia en éste de variables heterónomas que dispersan su control, dinámica que no solo se manifiesta entre campos de conocimiento, por ejemplo, el de las ciencias sociales o humanas, respecto al de las ciencias matemáticas o biomédicas, sino también al interior del campo. En tal caso, la vulnerabilidad heterónoma del Trabajo Social aumenta la capacidad de definición externa a la profesión acerca de lo que el Trabajo Social es y puede ser.

Por ejemplo, si observamos el tratamiento del Trabajo Social en clasificaciones oficiales del conocimiento encontramos el trabajo de Farías (El trabajo social y los campos disciplinarios de las ciencias sociales en Chile) que señala la invisibilidad del Trabajo Social como disciplina de las ciencias sociales en Chile, extendiendo su argumento a América Latina. J. Saavedra (La paradoja de la negación en la intervención social) identifica su omisión en ciertas definiciones operacionales sobre las ciencias sociales o de algunas taxonomías de organismos internacionales como la de la UNESCO-ISCED 2012. Asimismo, Lorente y Zambrano (2016) señalan que no puede obviarse que en la «Nomenclatura Internacional de la UNESCO para los campos de Ciencia y Tecnología» se considera una subdisciplina denominada Trabajo Social y Servicios Sociales, en el interior de Ciencias Económicas, bajo el descriptor "Organización Industrial y Políticas Gubernamentales" (código 5309.08). La pregunta es ¿cómo se determinó esta ubicación y por qué? Y cuál ha sido el trabajo hasta ahora de organizaciones como la FITS para abordar una situación como esta, ya que nos está ofreciendo una clasificación asumida institucionalmente en cualquier parte del mundo. Sin embargo, curiosamente, en Web of Knowledge las revistas de Trabajo Social se encuentran clasificadas en un apartado propio, lo que hace pensar que deben tener algún tipo de rentabilidad.

En la actualidad, la escasez de doctorados en Trabajo Social en América Latina y en España estimula que numerosas trabajadoras sociales se estén doctorando en programas de investigación que no recogen específicamente las inquietudes epistemológicas del Trabajo Social que, sin dejar de estar presentes, deben solaparlas metódicamente en sus trabajos de investigación. Ello conduce a un vaciamiento paulatino del capital de conocimiento propio que termina refundiéndose en otras disciplinas, mediante prácticas de apropiación, expropiación o centrifugación del conocimiento que aportan trabajadoras sociales. Los corolarios de ellos son serios, y no se le presta la debida atención. Por una parte, favorece la idea de que no se produce conocimiento propio, y por otra, se transfirieren a dichos posgrados datos de la primera línea de acción profesional que difícilmente se hubiesen conseguido si no fuese por el hecho de que quien investiga es trabajadora social. Estas prácticas de entrega y apropiación naturalizan la subordinación entre disciplinas, contribuyendo a la reproducción secular de formas de autoridad intelectual que ayudan a comprender la continuidad del componente subalterno en la identidad socioprofesional del Trabajo Social.

Mientras las disciplinas mejor situadas, a las que se le solicite tal relación de vecindad, no posean intereses específicos que coincidan con los de expansión del Trabajo Social, los efectos de este modo de proceder no tiene por qué incidir negativamente en la autonomía académica del Trabajo Social al interior de departamentos y facultades. Pero si los intereses se cruzan, la evolución académica y científica del Trabajo Social se encontraría hipotecada en una perversa economía del don, difícil de interrumpir en aquellas universidades donde la carrera exista. Unas y otras prácticas, si bien están motivadas por aspiraciones 
distintas, muestran las dificultades hacia procesos de autonomía en Trabajo Social, que vayan restando la pesada fuerza heterónoma a la que se ve sometido.

A su vez también se presenta el fenómeno de la seducción y encandilamiento que se gesta hacia otras disciplinas, lo cual es perfectamente comprensible cuando hay un interés profundo por el saber y por el avance de la investigación, pues es ahí donde se producen síntesis transdisciplinarias absolutamente necesarias. La dificultad aparece, en cambio, cuando la trabajadora social que investiga en el conocimiento de retorno, se le olvida admitir que parte de lo que depositó en su posgrado tiene que ver con las preguntas iniciales que se planteó o con las experiencias aportadas que surgieron del Trabajo Social.

Sobre ello se comentan dos propensiones identificadas, seguro haya algunas más. La primera es la que se observa en quien obtuvo su doctorado o magister, al regresar al espacio del trabajo social obvia que su conocimiento constituye una síntesis valiosa para seguir avanzando y que de ella participó el Trabajo Social. Sin embargo, opta por mantener que fue la otra disciplina la que le proporcionó en solitario su nuevo estatus académico, así haya extraído los datos de campo del ámbito profesional del trabajo social, que difícilmente hubiese conseguido si no fuese por el hecho de que es trabajadora social. Esto posee corolarios serios y poco atendidos desde la profesión, porque se transfieren a dichos posgrados información de la primera línea de acción profesional, dando lugar a una sustracción de los saberes acumulados del Trabajo Social, sin reconocimiento a la disciplina.

La segunda propensión que se apuntaba es, si cabe, más llamativa. Haber conocido otras disciplinas y haber disfrutado de las ventajas de un conocimiento más organizado, de un estatus más depurado y de mayor reconocimiento social, invita a un abandono abrupto o progresivo del Trabajo Social, aún las académicas en cuestión continúen teniendo responsabilidades docentes e investigadoras en la carrera de Trabajo Social. De quince entrevistas realizadas a colegas mujeres sobre este asunto, o sobre su desafección con el Trabajo Social tras realizar sus posgrados, señalan por orden las siguientes cuestiones: las condiciones para ampliar el conocimiento y generar investigación desde el Trabajo Social es precaria para continuar con sus carreras académicas; ser académicas al interior del Trabajo Social no está reconocido y es un esfuerzo poco útil, su posgrado le posibilitó disfrutar de otra posición y le permite "blanquear" su carrera, es decir, pudo librarse de prejuicios y de un estatus inferior en el mundo laboral; pudieron desempeñarse en mejores puestos académicos o profesionales. Este apartamiento o desafección curiosamente es expresada desde las mujeres entrevistadas como un proceso de reencuentro con condiciones más favorables a su desarrollo y con circunstancias menos duras de trabajo. Quizás haya que auscultar hasta qué punto las exigencias de subalternidad a las que restringe ser trabajadora social como identidad laboral están en concomitancia con las dificultades que imprime desenvolverse en una cultura del trabajo feminizada.

\section{La feminización de la profesión. Efectos en la construcción de una ciencia del Trabajo Social}

Desde que el Trabajo Social aparece como profesión, su composición ha sido mayoritariamente femenina, es decir, no ha experimentado una feminización sobrevenida, sino que es secular en relación con otras profesiones que ha incrementado en la última década el número de mujeres entre sus filas. La compilación de trabajos que Etzioni publicara en 1969 titulado The semi-professions and their organization: teachers, nurses, social workers es paradigmática, en principio porque solo la denominación ya señala el difícil camino que profesiones como el Trabajo Social han venido experimentando a lo largo del tiempo para ser reconocidas con un sentido diferente al que esta categoría sociológica, que acentúa el prefijo semi, lo constriñe. Las condiciones bajo las que opera una semi-profesión tienen que ver con experimentar una 
posición inferior en sus campos de conocimiento y acción, con desempeñar un rol de asistencia o servicios a otras, o en su caso, con recibir prescripciones de otras para actuar, y fundamentalmente, por no disponer de conocimiento abstracto que las sitúe en un orden de conocimiento jerárquicamente superior. Los corolarios que se derivan de tal conceptualización negativa advierten, según B. Lorente (2006), de la insuficiencia de aproximarse a la cuestión de la feminización desde su relevancia estadística, más que como resultado de como un proceso histórico y cultural de larga duración. De ahí que pueda postularse ingenuamente que equilibrar el número de activos, es decir, disponer de una cantidad similar de mujeres y hombres, puede reducir tales implicaciones subalternas.

La vinculación antropológica de las mujeres con la esfera del cuidado y sus derivaciones en la atención directa a las personas, no solo en el ámbito privado, sino también en el público proporcionará culturalmente un rasgo diferenciador a las profesiones secularmente feminizadas. Precisamente el cuidado social y la ayuda, aun cumpliendo una función económica y social imprescindible, está por fuera de las actividades percibidas como exitosas para progresar social y laboralmente. Desde luego, con ello no se sostiene que haya una predisposición esencialista hacia el cuidado y atención de otros en las mujeres, más bien apuntar que incluso en la actualidad, la posmoderna división sexual del trabajo, si es que puede llamársele así, se las apaña para reproducir cultural y simbólicamente un esquema del trabajo de larga duración en el tiempo, que sigue confinando fundamentalmente el cuidado y la ayuda directa en las mujeres, en tanto actividades nucleares de la reproducción social, desconociendo los nexos intrínsecos que producción y reproducción social mantienen necesariamente, incluso como biopolítica en la organización del trabajo de las sociedades contemporáneas.

Esta dinámica cultural feminiza un campo de conocimiento y también de actuación profesional afectando el desarrollo laboral y académico de las mujeres, y de los hombres que se encuentren en él, si bien el impacto de la feminización en unas y en otros no es coincidente. Ello da cuenta de hasta dónde el género es una construcción cultural en la que hombres y mujeres pueden socializar contenidos identificados como femeninos o masculinos, no obstante, el intercambio de papeles no es automático. Precisamente el que los papeles no sean fácilmente intercambiables, nos permite ver con mayor nitidez los conflictos de género al interior de profesiones feminizadas en trabajos como los de S. Fahlgren (The paradox of a genderbalanced workforce) y M. Peel (Male social workers and the anxieties of women's authority).

En esta profesión se identifica con nitidez la organización de la división social, científico-técnica y sobre todo sexualizada del trabajo, que muestra las intersecciones entre bajo estatus social, saberes aplicados y presencia femenina. El Trabajo Social constituye un extraordinario ejemplo para observar que la división del conocimiento es un hecho de poder que distribuye desigual y jerárquicamente el capital social asociado a los conocimientos considerados socialmente valiosos, procediendo a su reparto cultural de forma diferencial entre hombres y mujeres. Las identidades profesionales poseen una dinámica expansiva, pues así también garantizan su permanencia y reproducción a través del poder de definición e interpretación de la realidad social en la que interviene. Sin embargo, la presencia mayoritaria de mujeres en una profesión soporta mayores dificultades para sostener la autoridad sobre el proceso de producción, distribución y control del conocimiento propio que sustenta su campo profesional.

La menor valoración e incluso la invisibilidad secular de los aportes de las mujeres a la vida social es incuestionable actualmente, la extensa producción que el feminismo ha hecho en relación con ello, desde cualquiera de sus distintos enfoques paradigmáticos, deja poca duda empírica acerca de este hecho. EI mundo de la ciencia es uno de esos ámbitos dónde se pensaba que solo se equipararían saberes, sin intervenir el género de las personas que producían ciencia. Nada más alejado de la realidad, precisamente 
las aportaciones de las epistemologías feministas de Sandra Harding (Whose science? Whose knowledge? Thinking from women's lives) y E.F. Keller y H.E. Longino (Feminism and science) han sido reveladoras de las dificultades de las mujeres para acceder y mantenerse en el mundo científico. Desde enfoques plurales han objetivado el androcentrismo en la ciencia como un problema epistemológico y político de calado que atraviesa la naturaleza del conocimiento que produce la ciencia. Del mismo modo, han señalado la determinación del androcentrismo en el devenir histórico del conocimiento científico, subrayando los efectos y alcances de la racionalidad científica masculina, y de manera destacada han mostrado las vinculaciones entre sujeto cognoscente y la dimensión política y social del conocimiento; lo que contribuye a dejar en evidencia los nexos entre poder y ciencia. Estas contribuciones feministas han sido muy importantes porque cuestionan directamente el sesgo de género que posee la ciencia, que es un sesgo cultural que afecta a su axioma, pero también a sus conclusiones, sin embargo, la maquinaria científica y su racionalidad androcéntrica continúa funcionando y fagocitando las controversias de validez en este sentido.

Si el calado de estas discusiones se ha dirigido con énfasis al ámbito de las ciencias duras, con repercusiones en las ciencias sociales y humanas, quizás desde la envergadura que presentan estas discusiones sea perentorio para el Trabajo Social visualizar los contornos problemáticos en los que está situado. Desde una dimensión externa, para ser integrado como interlocutor válido al debate de las ciencias sociales en su lucha para ser reconocidas desde un paradigma empírico racional en el que se sostiene con dificultad. Desde una dimensión interna, para reconocer que el sujeto constructor de la disciplina adolece de una condición histórica y estructural feminizada que está impidiendo conocer con precisión los engranajes de discriminación a los que se ve sometido, como aquellos que termina reproduciendo al no haberse forjado una consciencia colectiva y reflexiva sobre el fenómeno, afectando consecuentemente a sus posibilidades de desarrollo como profesión y como disciplina. Precisamente investigaciones como la de M.J. Holosko (Citation impact of women in social work) dan cuenta de barreras estructurales que enfrentan las mujeres trabajadoras sociales en la academia, sin embargo, la cuestión de género en Trabajo Social, y por supuesto los corolarios de la feminización, aún tiene sus inconvenientes identificables en posiciones que controvierten la vigencia del feminismo en su interior como la de R. K. Caputo (What's epistemology got to do with it?) o que confrontan duramente la aplicación de las epistemologías feministas del punto de vista a los servicios sociales, en el caso de W. Epstein (The romantic retreat is not club med).

A estas alturas es un debate impostergable. Guste más o menos, plantear el problema de la ciencia en Trabajo Social, bien desde la posibilidad de una ciencia del Trabajo Social, bien desde su reconocimiento como ciencia social, requiere de la objetivación del sujeto constructor de la disciplina y en tal proceso asumir su feminización. Si la ciencia es una práctica social y cultural, las prácticas las llevan a cabo sujetos, y para el caso no una entidad abstracta que se llama Trabajo Social.

Este hecho es importante a tener en cuenta por el Trabajo Social, ya que mucha de su invisibilidad está relacionada con la identidad feminizada que permea sus prácticas, pero también con su campo de conocimiento y acción confinado simbólicamente más al terreno de la reproducción social, que a la de visibilizar sus conexiones reales e interdependientes con el de la producción social. Si como proponen las epistemologías alternativas, entre ellas, las epistemologías feministas, se pudiese trabajar en la objetivación práctica del sujeto cogsnocente del Trabajo Social, tendríamos una propuesta para visibilizar los caminos andados, pero también como han sido caminados. Ellas se concentran en varios ejes que harían posible comenzar con dicho proceso sobre la objetivación del sujeto feminizado del Trabajo Social. La primera de ellas tiene que ver con la relevancia del sujeto de conocimiento frente a un sujeto abstracto 
e ideal en la ciencia: el sujeto tiene un contexto en el que se hace, proporcionándole coherencia interna y externa, para el caso del Trabajo Social su formación se gesta en la larga duración. La segunda aboga por el carácter situado del conocimiento que confronta la noción asentada de objetividad. La tercera tiene que ver con la imposibilidad de mantener la neutralidad del conocimiento científico e identificar las relaciones de poder que tras dicha posición discursiva se sigue manteniendo

La necesidad de la emergencia del sujeto constructor de dichas prácticas de conocimiento y acción se torna en una condición ineludible, el resultado de ello puede ser cualquiera, con dificultad se prevén los caminos que un proceso de reconfiguración de dicha identidad laboral puede alumbrar, pero lo que no puede obviarse es la urgencia de llevarlo a cabo. El horizonte es un sujeto mejor posicionado, reflexivo y consciente de si, con un conocimiento más depurado sobre la relevancia de continuar demarcando y ampliando la importancia de los procesos de ayuda social y cuidado, con justicia, de lo que Seyla Benhabib llama el "otro" concreto.

La feminización es un hecho histórico que necesita ser enfrentado y demarcado en relación tanto a sus potencialidades, como a sus limitaciones, sin demagogias, entre otras, porque no es una casualidad la concentración en la primera línea de acción de tantas mujeres dedicadas a las actividades de protección social, y sobre todo a las directamente relacionadas con la pobreza, la vulnerablidad y el sufrimiento. Hay claramente una división del conocimiento y de la acción que está generizada, encarnada en un sujeto que ha producido saberes y prácticas históricas sobre estos campos de acción profesional que hoy forman una comunidad epistémica.

\section{Conclusiones}

A través de lo expuesto se identifica claramente la preocupación en la disciplina por el carácter científico de sus producciones, ya que sin teorías no podrían generarse preguntas, redefinir áreas de incumbencia de acuerdo con la complejidad y vertiginosidad de las últimas décadas, ni proponer abordajes acordes. Sin embargo, esta convicción relativamente consensuada enfrenta cadenas que erosionan la consolidación de una ciencia de una disciplina que lleva más de cien años buscándola. Algunas de estas cadenas son internas, derivadas de una posición dicotómica falaz entre teoría y praxis. Los saberes de la acción han sido largamente ignorados, fragmentados, sin instancias de interpelación ni diálogo que permitan dar un paso más allá de la descripción y articular teorías que necesariamente relacionan saberes producidos localmente. Insuficientes han sido las disposiciones para superar esto, el desdén hacia las prácticas consideradas de "escritorio" como la investigación, y que todavía en ocasiones es enfrentada como bandera del "conocimiento de élite" versus el "conocimiento de los territorios y comunidades". Tampoco ayuda que un bloque de la historia de la disciplina haya sido descartado por estar contaminado con el ideario "asistencialista cristiano" cuando lo que se necesita es continuar profundizando en las derivaciones económicas, éticas, políticas etc., que el cristianismo como hecho social e histórico ha supuesto no solo en el pasado, sino incluso en la actualidad, tras su secularización, en el desarrollo de la acción social en su conjunto. Otro aspecto que mencionar son los saberes ignorados, no ya a propósito como en el punto anterior, sino porque en algún momento de la trayectoria de la disciplina fueron "enajenados" reingresando a nuestro campo mediante disciplinas con mayor poder de definición para apropiárselos, darles sistematicidad y reorientarlos conceptualmente.

Pero el panorama no estaría completo si no se incluyen los condicionantes externos que aparecen cuando el análisis incluye la posición que la disciplina tuvo en la estructura de la sociedad. La invisibilidad de los saberes dinamiza las luchas de las disciplinas por recortar espacios como propios, pero también enajenan 
y debilitan fuerzas, entre otras, porque los límites siempre porosos y cambiantes de las fronteras disciplinares, no todas con idéntica jerarquía e igual poder definición, coadyuvan prácticas subalternas y vulnerables en las relaciones de conocimiento, académicas y profesionales.

La autonomía como disciplina científica depende de las comunidades epistémicas que conviven en Trabajo Social puedan hacer explícitos sus debates, que no implica solamente revisar elementos epistemológicos, sino que está también vinculado a las relaciones con otras ciencias y contextos disciplinarios, a las concepciones del mundo que se reproducen desde el seno de la disciplina, la axiología, es decir, a los valores del campo y a los modelos de ética en los que encuentran sentido, tales como la ética del otro concreto de S. Benhabib (EI ser y el otro en la ética contemporánea) que privilegia la equidad y la reciprocidad complementaria, o la ética ciudadana, que como nota Fóscolo para el Trabajo Social a partir de los desarrollos de Agnes Heller, se "comparta los valores y derechos de los co-sujetos como conciudadanos y acompañe las luchas de la ciudadanía por la emancipación" (Fóscolo 2006:178).

Se deben reconocer saberes sometidos, ausentes, subalternos en procesos de jerarquías en las que el Trabajo Social por cadenas internas y condicionantes externos no asentó visiblemente todavía un ethos disciplinar con reconocida capacidad, que conjuga más de un siglo de vida académica y en todas partes del mundo. Una descentralización epistemológica implicaría una apertura hacia otras formas de conocer, de representar y de representarse y que en nuestro ethos disciplinar conjuga tradiciones en prácticas de cuidado, en racionalidades de múltiples soportes que rescatan posiciones marginales como las de las comunidades rurales, pueblos indígenas, poblaciones estigmatizadas en las que valores como el cuidado son tan constitutivos de sus epistemes como aquellos que están soportados en luchas por el avance de la justicia social.

\section{Nota}

Por requerimientos del formato de la publicación, no fue incluida la totalidad de las referencias bibliográficas de los artículos que fueron utilizados para la confección de la matriz, los que podrán ser solicitadas directamente escribiendo al email de las autoras.

\section{Bibliografía}

Fóscolo, N. 2006. Desafíos éticos del trabajo social latinoamericano. Paradigmas, necesidades, valores, derechos. Buenos Aires: Espacio Editorial.

Foucault, M. 1986. Las palabras y las cosas. México: Siglo XXI.

Lorente, B. 2002. Trabajo social y ciencias sociales. Poder, funcionalización y subalternidad de saberes. Trabajo Social 4: 41-59. https://revistas.unal.edu.co/index.php/tsocial/article/view/32516

Lorente, B. 2006. Para una antropología del sujeto profesional en perspectiva histórica. La mujer y la ayuda social en el Occidente cristiano. Trabajo Social 8: 109-129.

https://revistas.unal.edu.co/index.php/tsocial/article/view/8501

Lorente, B. y Zambrano, C. 2016. El trabajo social en tiempos de apertura de las ciencias sociales, pp. 361-375. En: E. Raya y E. Pastor. Trabajo social, derechos humanos e innovación social. Pamplona: Aranzadi. 
Montaño, C. 2016. Pobreza, "cuestión social" y su enfrentamiento. Noesis 25(49): 69-98. doi: 10.20983/noesis.2016.1.3

Morin, E. 1994. Introducción al pensamiento complejo. Barcelona: Gedisa

Recibido el 16 Jun 2017

Aceptado el 25 Sep 2017 